\title{
Perceptions of factors influencing engagement with health and wellbeing apps: a qualitative study using the COM-B model and Theoretical Domains Framework.
}

\author{
Dorothy Szinay ${ }^{1}$, Olga Perski ${ }^{2}$, Andy Jones $^{1}$, Tim Chadborn $^{3}$, Jamie Brown ${ }^{2}$, Felix Naughton ${ }^{1}$ \\ 1 University of East Anglia \\ 2 University College London, University of London \\ 3 Public Health England
}

Funding: DS is funded through a PhD studentship, provided jointly by Public Health England and the University of East Anglia. OP receives salary support from Cancer Research UK (C1417/A22962).

Potential competing interests: JB has received unrestricted research funding to study smoking cessation from pharmaceutical companies who manufacture smoking cessation medications. JB, FN, OP and DS are unpaid members of the scientific committee for the 'Smoke Free' app and have no financial interest in the app.

\section{Abstract}

Objectives.

User engagement with health and wellbeing apps is typically poor. Understanding factors that influence engagement can inform the design of more engaging apps. This study explored users' experiences of and reasons for engaging and not engaging with health and wellbeing apps.

Methods.

UK-based adults $(\mathrm{N}=17)$ interested in using a health or wellbeing app took part in a semi-structured interview to explore experiences of engaging with these apps. Data were analysed with the framework approach, informed by the Capability, Opportunity, Motivation - Behaviour (COM-B) model and the Theoretical Domains Framework, two widely used frameworks that incorporate a comprehensive set of behavioural influences.

\section{Results.}

Factors to influence capability included accessible information (e.g. user guidance, statistical and health information), reduced cognitive load, well-designed reminders, self-monitoring features, features that help to establish a routine, features that offer safety netting and stepping-stone app characteristics. Tailoring, peer support and embedded professional support were identified as important factors that enhance users' opportunities for engagement. Feedback, rewards, encouragement, goal setting, action planning, self-confidence and commitment were judged to be motivation factors that affect engagement.

\section{Conclusion.}

Multiple factors were identified across all components of the COM-B model that may be valuable for the development of 
more engaging health and wellbeing apps. Engagement appears to be influenced primarily by features that provide user guidance, promote minimal cognitive load and support self-monitoring (capability), provide embedded social support (opportunity), and goal setting with action planning (motivation).

\section{Introduction}

Smoking, physical inactivity, inadequate diet, and excessive alcohol consumption are the main risk factors for noncommunicable diseases, responsible for over 56.9 million deaths worldwide [1]. People with mental health problems often have poorer physical health and vice versa $[2,3]$. To reduce the burden of ill health, a range of interventions have been developed. Integration of multimedia technologies within the healthcare domain has led to the development of interventions delivered digitally via mobile phones, wearable devices and smartphone applications ('apps'). Smartphone apps are constantly available to the user and therefore act as portable tools for the delivery of easily accessible health and wellbeing interventions [4]. There is early evidence of effectiveness of apps for physical inactivity [5-8], weight loss [7,9,10], alcohol reduction in non-dependent drinkers [11] and mental health promotion [12]. Health apps are also considered a cost-effective solution $[7,13]$ and have the potential to increase access for hard-to-reach populations that are resistant or unable to seek face-to-face support, for instance due to stigma or geographical barriers [14].

Engagement is a necessary component for the effectiveness of a health or wellbeing app. Engagement with health and wellbeing apps can be defined as '(1) the extent (e.g. amount, frequency, duration, depth) of usage and (2) a subjective experience characterised by attention, interest and affect' [15]. Despite the promise of health apps, engagement tends to be poor [16,17]. For example, a Mobile Consumer Report found that for medical, health and fitness apps, only $20 \%$ of users use the app one day after installation, and only $8 \%$ after seven days after installation [18], A panel-based analysis systematically examined usage patterns in 93 mental health apps and found that the median app retention rate at 15 and 30 days after installation were $3.9 \%$ and $3.3 . \%$, respectively [17].

There is a growing literature on factors influencing engagement with health and wellbeing smartphone apps. In our recent review of 41 studies, we identified 26 factors important for the uptake of and engagement with such apps [19]. In addition to a wide range of behaviour change techniques (e.g. self-monitoring, goal setting) $[20,21]$, several other factors were identified as influential, including the role of healthcare professionals in the promotion and recommendation of health apps [22] and embedded professional support [23]. The latter was found to be particularly important for certain behaviours (i.e. alcohol reduction, suicide prevention, anxiety, self-harm), with stand-alone apps considered insufficient by users and clinicians [14]. In an assessment of 93 mental health apps, daily minutes of engagement were higher for apps that included peer support (median=35.1, IQR=N/A, $n=2$ ) and coping strategies, such as mindfulness and meditation (median=21.5, $I Q R=15$ ) compared with apps that incorporated self-monitoring or psychoeducational features (median range=3.538.32) [17]. Few qualitative studies have been undertaken to explore factors that affect engagement with health and wellbeing apps. Those undertaken have focused on specific populations or behaviours. Available studies have focused on weight loss behaviours and alcohol reduction, and have found that health information provided [16,21], personalisation of app content [24] and tailoring of content to the user's demographics [21] are some of the factors deemed to be important for 
engagement with weight loss and alcohol reduction apps. Most studies conducted to date investigate features of health apps that are desirable by a certain population, and little is known about factors deemed important for engagement with a wider range of health and wellbeing apps.

\section{Theoretical framework}

The COM-B (Capability, Opportunity, Motivation - Behaviour) model [25] provides a broad framework for understanding factors influencing user engagement. According to the COM-B model, behaviour (e.g. app uptake and engagement) arises from the interaction between the individual's physical (e.g. app skills) and psychological (e.g. knowledge of using an app) capability, physical (e.g. features of the app) and social (e.g. recommendations for an app) opportunity, and automatic (e.g. feedback received from an app) and reflective (e.g. user's self-confidence of using an app) motivation. The Theoretical Domains Framework (TDF) [26] is a synthesis of 33 theories and 128 psychological constructs, and includes 14 domains that can be mapped under the three main components of the COM-B model. Taken together, the COM-B and TDF provide a detailed theoretical framework that allow the careful consideration of factors influencing engagement with health and wellbeing apps (see Figure 1).

\section{Aim}

We aimed to apply a theoretical framework informed by the COM-B model and the TDF to investigate people's experiences and reasons for engaging and not engaging with health and wellbeing apps using qualitative interviews. The findings may inform future app development to improve user engagement. 


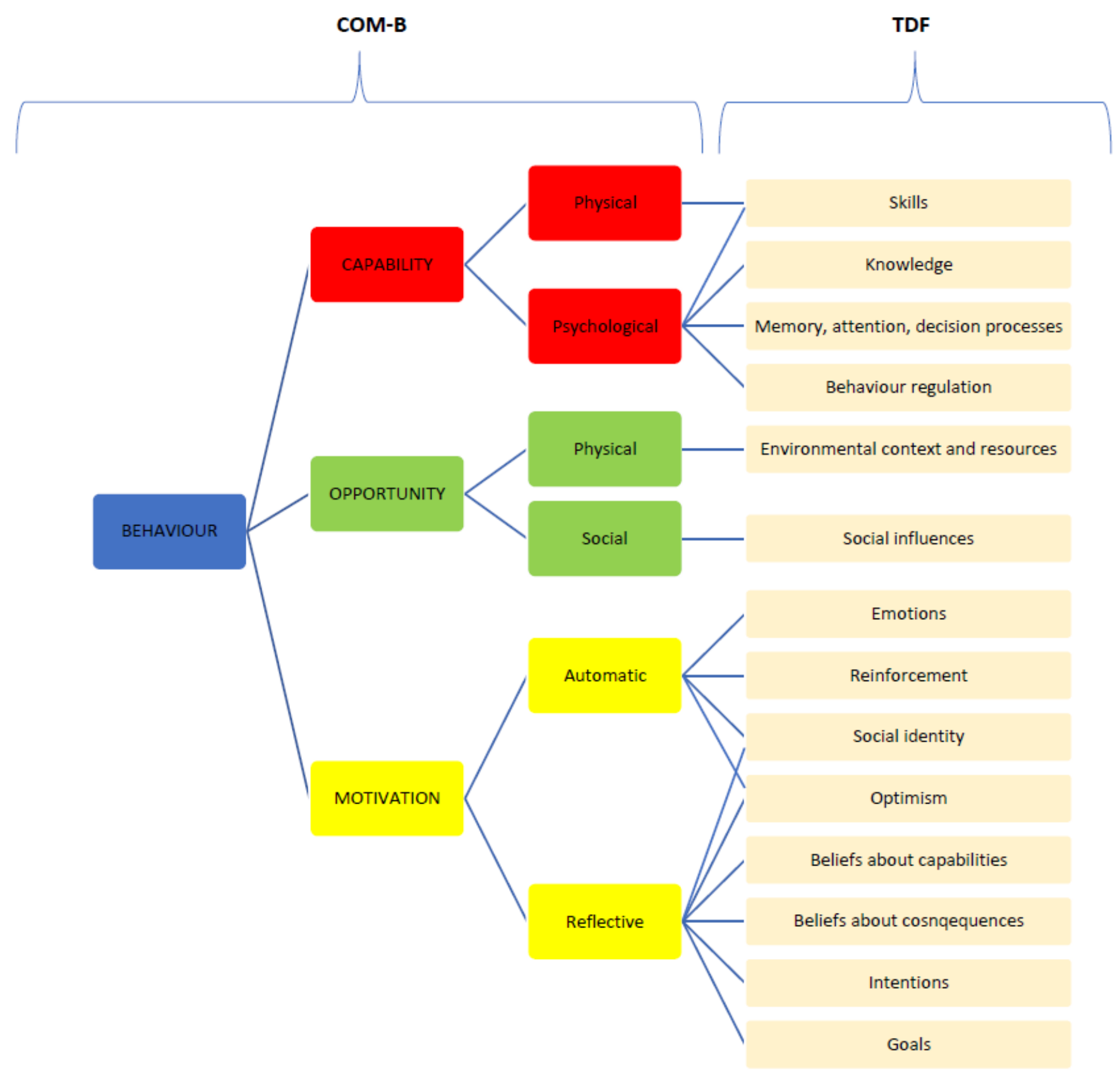

Figure 1. A visual representation of how the Theoretical Domains framework can be mapped onto the components of the COM-B model [19].

\section{Methods}

\section{Study design}

This qualitative study used semi-structured interviews [27], and was designed and reported in line with the Consolidated Criteria for Reporting Qualitative Research (COREQ) checklist [28]. The study protocol was pre-registered on the Open Science Framework (https://osf.io/jirkd3/). This study was part of a larger project investigating both the uptake of and engagement with health and wellbeing apps. 


\section{Participants and recruitment}

Participants were recruited through social media. Recruitment through Facebook is known to be an effective way to reach adults interested in health and wellbeing apps [29]. It is fast and cost-effective, and has been found to provide better representation and improved participant selection compared with traditional recruitment methods [29]. Eligible participants for this study 1) were aged 18 years or over; 2) were able to give consent; 3) owned a smartphone; 4) would consider using a smartphone app to change their behaviour in the future; 5) could travel for an interview. Purposive sampling was used to ensure diversity of the sample (age, gender, ethnicity, educational level, employment) [30].

\section{Measures}

To determine eligibility and to describe the sample, data were collected on 1) age, 2) gender, 3) ethnicity, measured using the Office for National Statistics' index, 4) level of education, 5) employment status, 6) whether they had ever used a health or wellbeing app, 7) whether they currently use a health or wellbeing app, 8) last time they downloaded an app, and 9) frequency of app use.

\section{Procedure}

Participants read the information sheet available online. Once read and expressed interest to participate, participants filled in an online questionnaire to assess their eligibility and to collect descriptive data (Additional file 1.). The questionnaire was hosted by 'Jisc Online Surveys' software. Participants meeting the inclusion criteria received a comprehensive participant information sheet via email and were invited for an interview. On the day of the interview, interviewees received a printed copy of the participant information sheet, and written consent was obtained.

The interviews were conducted by a female researcher (DS) between July and August 2019. The interviews took place face-to-face in Norwich, England, at the University of East Anglia $(n=17)$ or in the participant's home $(n=1)$. One participant was not included in this study as they had no previous experience of using health or wellbeing apps. No one else was present during the interviews. Sessions lasted between 26 and 63 minutes. Semi-structured interview techniques were used to elicit data on participants' experiences of and views on engagement with health and wellbeing apps. The final topic guide was informed by feedback from key stakeholders, including patient and public involvement representatives and domain experts from Public Health England. A think aloud task (reported elsewhere) was followed by several questions regarding participants' experiences of engagement with apps (see Additional file 2 for the topic guide). Participants received a £20 gift voucher as compensation for their time.

\section{Data analysis}

The interviews were audio-recorded and transcribed verbatim. Data were analysed using framework analysis following the stages of familiarisation, identification of thematic framework, indexing, charting, mapping and interpretation [31]. A percentage of randomly selected transcripts (15\%) were independently coded by the second author (OP). Through repeated discussions between the first and second authors, the deductive thematic framework based on the TDF was refined iteratively. Any discrepancies were resolved through discussion with a further author (FN). Indexing was completed 
by DS using QSR NVivo 12 (REF). Indexing was followed by charting, and the responses were grouped according to the finalised thematic framework. During mapping and interpretation, the grouped data were examined to identify patterns. Peer debriefing by the University College London Tobacco and Alcohol research group, with extensive experience of the application of the COM-B model and TDF in health research, was used to ensure the accuracy of data interpretation and data analysis. Peer debriefing is a form of analytical triangulation where researchers not directly involved in the study are prompted to provide input and critical opinions on various aspects of a project [32].

\section{External validity}

Member checking was conducted to ensure the trustworthiness of the results [33]. Six randomly selected participants (30\% of total sample) were contacted and invited to provide feedback on the summary of findings and the conclusion. The purpose of the member checking was to investigate whether participants agreed with the interpretation of the results and whether they felt their opinion was captured and appropriately presented. Two participants responded and indicated that they agreed with the interpretation.

\section{Reflexivity}

$\mathrm{DS}$, a PhD candidate, conducted the interviews after receiving extensive training in qualitative research methodology. The co-authors have experience in mixed-methods research and the application of the COM-B model and the TDF. Field notes and a research journal were kept during data collection. Efforts to establish good rapport with the participants was made throughout. The interview was individually adapted to the flow of discussion made by each participant. Most participants $(n=15)$ stated that they wished to find out more about the findings of the research.

\section{Ethical approval}

Ethical approval was obtained from the Faculty of Medicine and Health Sciences Ethics Committee at the University of East Anglia (Reference number: 201819 - 089). The collected data has been stored following the European Union General Data Protection Regulation (GDPR) and the University of East Anglia Research Data Management Policy. The data was anonymised, and all personal identifiers were removed.

\section{Results}

\section{Participant characteristics}

Eighteen adults (mean age $=43$, range 21-68) were recruited, of whom 10 were females, 14 were White British, 13 were employed full time, 8 had college degree or higher. Eleven participants reported currently using at least one health or wellbeing app at the time of the interview. Three participants expressed their intention to change one behaviour, with most participants interested in changing more than one behaviour (e.g. losing weight, being more active, managing their mood). One participant had never used health apps before; therefore, the findings of this paper are based on the views and experiences of the remaining 17 participants about their engagement with health and wellbeing apps (see Table 1). 
Table 1. Participants characteristics

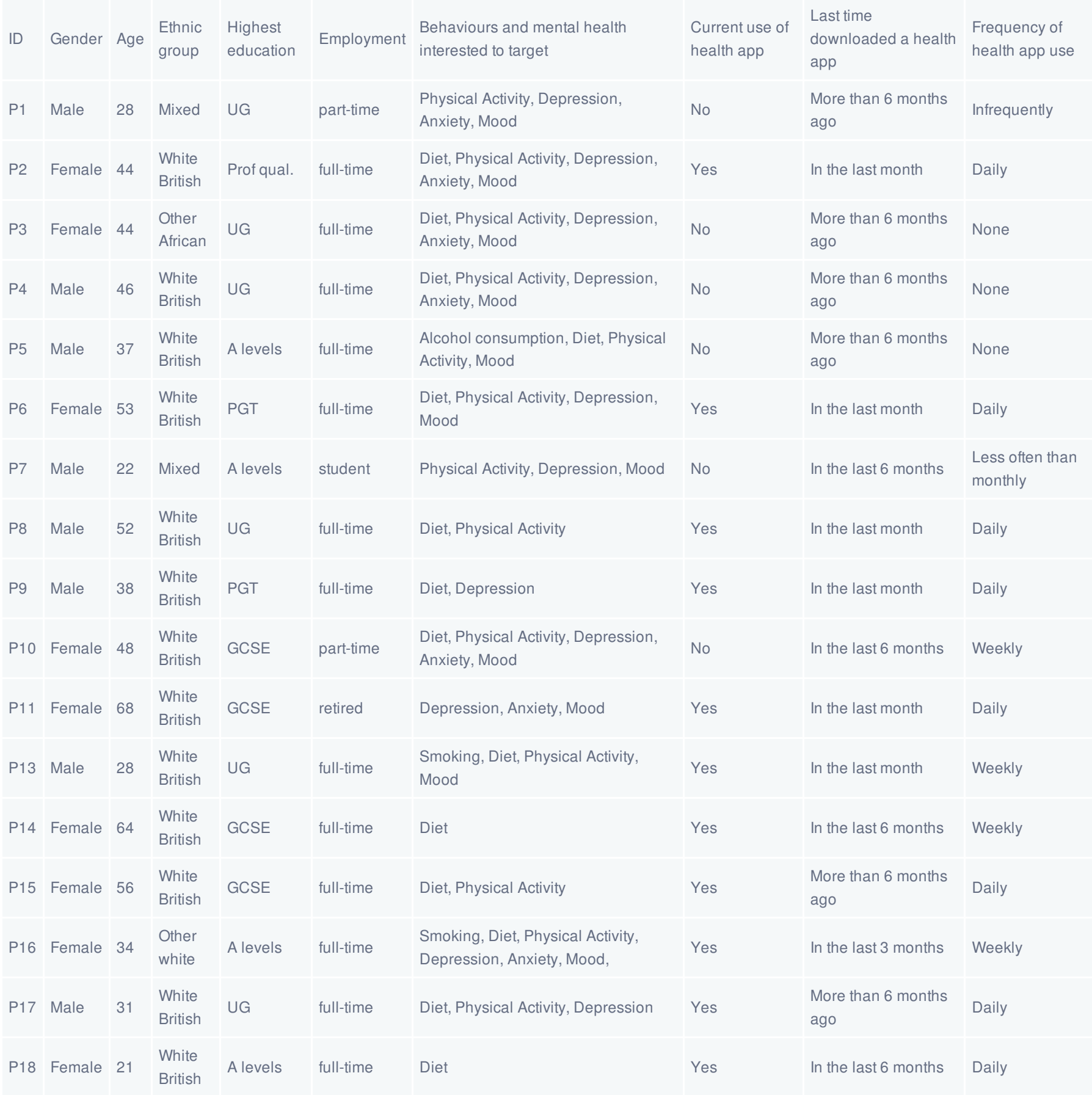

Note: GSCE - General Certificate of Secondary Education (in the UK), A levels - General Certificate of Education Advanced Level (in the UK); UG - Undergraduate Degree; PGT - Postgraduate Taught Degree

Factors influencing engagement with health and wellbeing apps

An overview of the factors mapped under the constructs of the TDF and components of the COM-B can be found in Table 2.

Table 2. Perception of factors influencing engagement with health apps 


\begin{tabular}{|c|c|c|c|}
\hline $\begin{array}{l}\text { COM-B }^{\mathrm{a}} \\
\text { component }\end{array}$ & TDF $^{\mathrm{b}}$ construct & Factors & Description \\
\hline \multirow{9}{*}{$\begin{array}{l}\text { Psychological } \\
\text { Capability }\end{array}$} & \multirow{3}{*}{ Knowledge } & User guidance & - Instructions on how to effectively use a health app \\
\hline & & $\begin{array}{l}\text { Statistical } \\
\text { information }\end{array}$ & - A visual or numerical summary of progress or quantification of the behaviour \\
\hline & & $\begin{array}{l}\text { Health } \\
\text { information }\end{array}$ & - Educational information related to health and wellbeing aspects \\
\hline & \multirow{2}{*}{$\begin{array}{l}\text { Memory, attention } \\
\text { Decision } \\
\text { processes }\end{array}$} & $\begin{array}{l}\text { Reduced } \\
\text { cognitive load }\end{array}$ & - The app is not too time consuming, easy to use and requires minimal input \\
\hline & & Reminders & - Preferably customisable, notification-type messages \\
\hline & \multirow{4}{*}{$\begin{array}{l}\text { Behaviour } \\
\text { regulation }\end{array}$} & Self-monitoring & - The ability of the app to support self-regulation of the target behaviour \\
\hline & & Routines & - The ability to support routine/habit formation \\
\hline & & Safety netting & - Retaining the app for a potential precipitating event in the future \\
\hline & & 'Stepping stone' & - App as a first step in the behaviour change process \\
\hline $\begin{array}{l}\text { Physical } \\
\text { Opportunity }\end{array}$ & $\begin{array}{l}\text { Environmental } \\
\text { resources }\end{array}$ & Tailoring & $\begin{array}{l}\text { - Innovative features and adaptability, and an interactive, two-way communication between the app } \\
\text { and user }\end{array}$ \\
\hline \multirow{2}{*}{$\begin{array}{l}\text { Social } \\
\text { Opportunity }\end{array}$} & \multirow[t]{2}{*}{ Social influences } & Peer support & $\begin{array}{l}\text { - Including social interaction with users with similar needs within the app or within their community; } \\
\text { a choice to connect to social media platforms, competitions and challenges with others or with } \\
\text { themselves }\end{array}$ \\
\hline & & $\begin{array}{l}\text { Social support } \\
\text { (practical) }\end{array}$ & - Possibility to contact health professionals and practitioners within the app \\
\hline \multirow{4}{*}{$\begin{array}{l}\text { Reflective } \\
\text { Motivation }\end{array}$} & $\begin{array}{l}\text { Beliefs about } \\
\text { capabilities }\end{array}$ & Self-confidence & - Perceived capability to change one's behaviour using an app \\
\hline & \multirow{2}{*}{ Goals } & Goal setting & - Establishing what the user would like to achieve \\
\hline & & Action planning & - Establishing how the user would like to achieve set goals \\
\hline & $\begin{array}{l}\text { Beliefs about } \\
\text { consequences }\end{array}$ & Commitment & $\begin{array}{l}\text { - The level of commitment while engaging with an app to change the behaviour and achieve set } \\
\text { goals. }\end{array}$ \\
\hline \multirow{6}{*}{$\begin{array}{l}\text { Automatic } \\
\text { Motivation }\end{array}$} & \multirow{3}{*}{ Reinforcement } & Feedback & - Feedback regarding the user's performance \\
\hline & & Rewards & $\begin{array}{l}\text { - Tangible (objects, discount, etc.) and intangible (badges, certificates, etc.) rewards in response to } \\
\text { the user's effort } \\
\text { - Gamification elements }\end{array}$ \\
\hline & & Encouragement & - Additional ways to provide reinforcement (e.g. encouraging messages) \\
\hline & \multirow{3}{*}{ Emotions } & $\begin{array}{l}\text { Positive } \\
\text { emotions }\end{array}$ & $\begin{array}{l}\text { - Triggered by included user guidance, statistical information, additional health information, } \\
\text { embedded professional support, community networking possibilities, tracking features and } \\
\text { rewards }\end{array}$ \\
\hline & & $\begin{array}{l}\text { Negative } \\
\text { emotions }\end{array}$ & $\begin{array}{l}\text { - Triggered by lack of user guidance, invasive push-notifications, cognitive overload, unrevealed in- } \\
\text { app costs }\end{array}$ \\
\hline & & Mixed emotions & - Triggered by reminders \\
\hline
\end{tabular}

${ }^{a}$ COM-B: capability, opportunity, motivation, behaviour model.

bTDF: theoretical domains framework.

Capability to engage with health and wellbeing apps 


\section{Knowledge}

Many participants perceived their knowledge on how to use an app, as well as embedded statistical and health information, as an important influence on their engagement with an app. We inferred this from the desire many people reported for clear user guidance and, in some cases, for help on how to increase their capability to perform a behaviour (e.g. demonstration of the behaviour). One participant explained that they had stopped using an app in the past due to there being "insufficient guidance on how to use it." (P8)

So this is where I start getting, well why are you asking me these questions if you're not going to let me carry on with it and that's where I start getting confused, going back, not really understanding where I need to go from here." (P15)

Further, the necessity of statistical information about their progress and achievements was reported by most participants:

"It's nice to see your progress on a graph and it's just very clear. It's a single screen, you have icons for all the activities that you've done during the day." (P6)

In addition, most participants expressed the need for relevant and comprehensive health information.

“Knowledge is key."(P14).

Several participants stated that having educational articles embedded would help them to build knowledge, and to understand and to manage their behaviour better. Not getting enough health information was reported as the main reason for one participant to look for a different app.

"It's got to have the information that I want and have it easily accessible." (P2)

\section{Memory, attention, decision processes}

Participants perceived reduced cognitive load and customisable, notification type reminders as factors that positively affect their capability to engage with an app. All participants described favouring apps with reduced cognitive load. This included apps with limited complexity, less data input, and a limited number of available features to choose from.

One participant suggested that an app should apply a multi-level approach with "a light version of an app and then enhanced"(P15). They described that an app might have a simple version for basic users with no registration and minimum data input and a more advanced version with all features available for power users.

Several participants expressed that a time-consuming app would be immediately deleted.

"A mood tracker is something I probably wouldn't use because it looks like it would require a lot of data of me putting in and typing it on to stuff." (P7) 
Although push notifications were considered more or less annoying, many participants described reminders as being particularly useful. One participant described that not being reminded to engage with an app led him to disengage.

"Because I wasn't reminded, I stopped using it. And I think that's really important." (P1)

However, a few participants who reported not finding notifications useful stated that they would immediately turn reminders off or delete the app.

"I'm sure there are many apps l've deleted because of reminders." (P7)

Others suggested that reminders might cause harm. For example, one participant described uninstalling a smoking cessation app as reminders were periodically reminding them about their addiction, thus serving as a prompt that induced cigarette cravings. Two participants proposed that opting in to receive reminders would be desirable instead of opting out. In addition, one participant suggested that human-like reminders in the form of text messages would be less likely ignored, and would create the perception of a human touch within the app.

“I think text messages would work better because I don't ignore my text messages and my WhatsApp messages because there's real people connected to those, you know? (...) if I could think of an ideal it would be a text message that kind of asked you a question and you replied and it felt like it was a human being." (P6)

\section{Behaviour regulation}

Participants perceived that self-monitoring, established routines, as well as safety netting and 'stepping-stone' characteristics of the app, would enhance their engagement with an app.

All described self-monitoring features as key in behaviour regulation, even when there is no particular goal set or when achieving the goal shows a delay.

"Monitoring, really because the goal is probably going to go a bit by the wayside because work has been too busy and life has changed and lots of stuff has happened this year. So l'm behind my goal but I still use it as a monitor." (P17)

Some participants reported that a daily routine of using an app would make engagement with it more accessible and continuous. Two participants described how using a weight management app for a week was necessary for them to get into a routine and helped them staying engaged after that. However, one of them explained that it felt difficult using the app at the beginning, although after a few days it got easier.

A number of participants explained they perceived physical activity apps as stepping stones to physical activity services with the app acting as an intermediate tool in behaviour change. Two participants described that an app helped them to get 
enough experience and practice home-workouts that boosted their confidence to sign up for a gym membership eventually.

"You can just literally do it at home [fitness app] until you feel I suppose a bit more confident to go out and join [the gym]." (P10)

Many of the participants described apps as a safety netting tool (e.g. relapse prevention). Several reported a tendency to re-engage with a weight management app periodically and when necessary to regulate their weight, for example before or after a holiday season, or an important upcoming event because the app had helped them achieve their goals in the past.

"I think I have periodically come back to it and thought 'no it worked before, it'll work again'." (P13)

Opportunity to engage with health and wellbeing apps

\section{Environmental resources}

Participants perceived that tailoring the technology was a factor that would influence sustained engagement. Many participants expressed the need for features that would create a better physical opportunity to engage with an app, and a more personalised experience during the engagement. Many participants described seeking to engage with apps that provide two-way communication that can adapt to the person's needs based on how they interact with such tools. Several participants mentioned the inclusion of innovative features. These features consisted of embedded artificial intelligence to receive health-related advice and tailored content, facial recognition and recognition of non-verbal cues for better outcomes in physical activity, e.g. correcting posture, and using the phone's camera for providing nutritional data of cooked food.

"If it's smart, as well. Has it got a little bit of artificial intelligence built into the background? Is it using my data? Is it saying "do you know what? Actually, you've done really well this week, you've used the app this amount of times. How are you feeling?" (P2)

One participant described that the lack of novelty of an app would lead them to disengage with it. In contrast, another reported the opposite; they would feel put off if they would need to learn new features.

"It's no good downloading an app and then six months later looking at that app and it's still the same, that would stop me." (P14)

'If something's working we want it to stay as it is, we don't want it to change, and even if there are improvements to it, if it's new it can kind of put people off in a way." (P13)

Syncing with wearables or other additional devices was described as desirable by many. 


\section{Social influences}

Peer support and social support (practical) were perceived by participants as factors that may sustain engagement with an app. Several participants perceived networking within an online community necessary peer support. Some described that sharing and exchanging experiences with others would encourage and motivate them in their journey. Others suggested anonymity for users as well as a moderation of discussions to avoid "misinformation" (P12).

"I like the idea that it's round the clock support, because so very often with mental health issues it's kind of 2 o'clock in the morning that they are the worst, and that is when you need to talk to somebody, and the idea of having a community who you don't have to explain how you're feeling sounds really good." (P11)

Embedded social media to share their progress with others was reported useful feature only by a few participants who were using physical activity or weight management apps. However, a couple of participants highlighted that this feature should be optional. Physical activity and weight management app users also described challenges and competitions as motivating and fun:

"There's challenges, which will help you with your weight loss, your fruit and vegetable intake, the exercise challenges that you can do, either with yourself or your friends, which are good for motivation." (P15)

All participants expressed their preference for an app that would offer built-in professional support, such as health practitioners, coaches and dieticians (social support, practical). One participant with an existing medical condition described the need for health practitioner support within an app. Additionally, two participants described that built-in support would help with accountability, and one participant indicated they would be willing to pay to access an app with inbuilt support. Another participant commented that the embedded professional support was the best feature of a mental health app they were using:

"Yeah if you could sort of talk to a healthcare professional in that app I think that would be better, because then they would have the up to date I suppose treatments and methods so that you know you're not going on old information." (P10)

"I: If you would need to say just one thing that is the best in the app, what would that be? P: The support." (P11)

Motivation to engage with health and wellbeing apps

\section{Beliefs about capabilities}

Apps were perceived by several participants as useful tools to enhance their self-confidence in changing their behaviour. One participant described that the community networking opportunities further helped her self-confidence and motivated her to use the app:

"The app made me feel more confident in doing it, even it was just basic home exercises." (P7) 


\section{Goals}

Goal setting and action planning were perceived as key factors for sustained engagement and motivators of behaviour change. Goal setting was reported to be valuable by all to address behaviour change, but half of the participants described the need for action planning features to help them achieve their set goals:

"I'd want something which was a bit more than press one button every day to say you haven't smoked; it was great for the first 10 minutes of using the app because I got all this information about 'wow thousands of pounds and the health benefits', and then after that it was literally just press this button to say you haven't smoked, and that wasn't really enough for me." (P13)

\section{Beliefs about consequences}

Several participants expressed that their level of commitment to achieve their goal shaped the level of engagement with the app they used:

"The app, the initial - the main reason you're on that app is to get your result of what you want to achieve, what you want to do to help you stay on track." (P9)

\section{Reinforcement}

Many participants perceived feedback, rewards and encouragement automatic motivational factors that may sustain engagement with an app. A number of participants expressed that they needed continuous feedback to reinforce their continuous use:

"I think an app that might give you feedback, a notification, that would keep me entertained and would keep my level of focus and wanting to continue with it." (P3)

Intangible rewards (i.e. badges, certificates) were described as another form of reinforcement by several participants, for motivating them and as "nice" (P14) or something to "show off" (P5). However, some other participants described intangible rewards as "irrelevant". They reported that the tangible rewards they received in the past including cinema tickets, lower insurance premiums, loyalty points that can be exchanged for objects or a free water bottle, provided better motivation to engage with the app than intangible ones. In addition, a few participants expressed the need for encouragement in the form of motivational messages:

"In this context, so badges, you earn nine of 24 badges so far. For me a little bit irrelevant actually, what are you going to do with it, there's other reasons why you're quitting, not to get the badges." (P16)

\section{Emotions}


Participants expressed positive emotions regarding available user guidance, statistical information, additional health information, embedded professional support, the possibility for community networking, self-monitoring features and rewards. However, negative emotions were expressed by lack of user guidance, invasive push-notifications and cognitive overload. Finally, reminders were person dependent and triggered mixed feelings across participants.

\section{Discussion}

\section{Principal findings}

This study applied the COM-B and the Theoretical Domains Framework (TDF) to explore users' views about factors that influence engagement with health and wellbeing apps. We found that knowledge, such as user guidance and statistical information, memory, attention and decision processes, such as reduced cognitive load, environmental resources, expressed by the tailored technology, and social influences, referred as peer and professional support, are most important factors for these participants for engagement.

This research is the first to identify the perception of certain apps as 'stepping stones' to more intensive behaviour change. For example, a home-based workout app or a walking app could seek to provide enough self-efficacy and competence for an individual to join a gym or start using a running app. An explicit 'stepping stone' approach could be a useful addition for apps targeting behaviours that are harder to achieve because of negative emotions, such as embarrassment, shame or pressure, including those targeting sedentary behaviour.

Many factors that were identified in this study are consistent with previous literature. Previous research found that engagement with health apps is greatly influenced by factors affecting users' capabilities including different types of knowledge (user guidance, statistical information, health information) [19,34], reduced cognitive load, reminders and selfmonitoring features $[19,21,35]$. These factors could be targeted during app development updates of existing apps to improve user engagement. In line with previous findings, reminders were not found to be universally useful [19]. One possible explanation is that reminders may be behaviour-dependent and person-dependent. Some participants reported that they had stopped engaging with a health app because they were not reminded to continue using it, while others tended to ignore or delete apps that sent reminders.

Engagement is further influenced by users' physical opportunities, such as tailored technology, and social opportunities, peer support including community networking, embedded social media and social competitions, and professional support $[19,23,24,35]$. Some users would want the app to be based on machine learning opportunities and on two-way interaction with users. The adaptable nature of an app and the provision and level of artificial intelligence (Al) included may also play a key feature in engagement. These factors may be harder to include once an app is developed; therefore, it might be important to consider these aspects in the development process. Indeed, such tailored technology may be the most important aspect to consider. For example, while there may be financial considerations precluding the provision of personal professional support within an app, this service may be developed using Al. These forms of technological 
personalised models in health behaviours such as nutrition or smoking, including machine learning models, has been suggested to aid the process of making decisions about diet and food [36]. However, Al was not yet found in diet monitoring apps [37]. A randomised controlled trial found that participants allocated to an advanced version of a smoking cessation app with an Al chatbot had 107\% higher engagement with the app, and over twice the odds of being abstinent at one month follow up, compared with participants using the standard version of the app [38]. Furthermore, timely Al-based behaviour change support received just-in-time may further increase behaviour change. Although unguided interventions can be effective, having professional support within an app tend to increase effective engagement [39]. Simple interventions that does not require professional support can be more widely disseminated and are more cost-effective than those with embedded professional support [39].

Users' reflective motivation, including beliefs in their capabilities (self-confidence) and consequences (commitment) as well as goals (goal setting and action planning), are essential for engagement. While the first two are harder to address because these are within-person factors, the latter could be easily implemented as features of the app. One possible way to increase self-confidence and commitment is perhaps to address these within the app by using quizzes or articles [40], (e.g. for commitment 'How to stay on track to achieve your goal?') or check-in messages using Al [38].

Emotions are considered automatic motivation factors and are a powerful driver of behaviour that affect adherence, for example engagement with a health app [41]. It is noteworthy that we did not identify emotions directly influencing engagement or we failed to identify them. However, we found evidence that the other factors affected participants' emotions. Appealing features, such as statistical and health information, embedded peer and professional support, and tracking features and rewards, triggered positive emotions. In contrast, lack of user guidance, invasive notifications and cognitive load triggered negative emotions. A better understanding of how the presence or absence of specific features affect participants' emotions may be useful for the development of new or refinement of existing apps, which, consequently, may lead to better engagement with health apps.

\section{Strengths and Limitations}

A strength of the study is the methodology used. First, to assure that the research was as relevant and meaningful as possible, the study protocol was developed with policymakers and patient and public representative involvement in the design of the topic guide. Second, the research was further informed by well-established theoretical models; the COM-B and the TDF and peer debriefing was used to help the data interpretation and data analysis [32]. Third, the purposive sampling technique allowed the recruitment of a diverse sample regarding their gender, educational level and employment status. Finally, member checking was conducted, a technique used to establish the credibility of the findings by sending a brief summary of it to randomly selected participants [33].

The study has several limitations. First, the recruitment of a sample of participants with more diverse demographics might have identified additional factors that are important for engagement. Second, out of six participants contacted, only two 
responded to the request for external validation. Therefore, it is not clear whether the other participants disregarded our request or disagreed with our interpretation.

\section{Implications and future research}

This research provides insight for stakeholders in public health, policymakers, and developers of apps that target disease prevention and health promotion. Our findings may also be used to inform the development of interventions aiming to promote engagement with evidence-based health and wellbeing apps. In the UK, this aligns with the priorities of the National Health Service's the NHS Long Term Plan (i.e. 'digital first').

Our main finding is centred around providing necessary support for increased engagement with health apps. We found that embedded professional support may have a substantial impact on engagement, although it may not be beneficial for all health behaviours. Embedded social support may be particularly important for some behaviours that are more likely to be complex and require intensive support in order to maintain engagement. These behaviours are the ones that require reassurance, guidance or emotional support [39], such as apps targeting substance misuse, or the ones developed to improve mental health. While it is not always feasible to develop an app with embedded professional support, there might be different ways to address this need outside of the app. For instance, there may be a way to provide support within the community-based care to assist with the uptake of health apps and with the progress or potential barriers of engagement. Another way to mitigate the absence of embedded professional support is to investigate the potential efficacy of advanced computational techniques, such as Al, to mimic the support provided by healthcare professionals (e.g. in the form of chatbots or other types of conversational agents). There is an urgent need for more research on the optimal type (e.g. technology-mediated or 'blended') and timing of support needed within various health and wellbeing smartphone apps.

To better meet users' needs, the design of apps would ideally be informed by a user-centred and iterative development process, supported by mixed-methods research including in-depth interviews. As app engagement is generally greater in those with higher socioeconomic status [42], involving individuals with lower socioeconomic status is particularly important [39]. Furthermore, people directly affected by the digital divide, or digital exclusion and who may struggle to benefit from health apps due to a lack of skills or low digital literacy, could be targeted by offering app-use tutoring. While this may require investment or relocation of resources within community health care settings, it may increase the reach of health apps and lead to a greater public health benefit.

Additionally, more experimental research would help us to better understand the effects and potential interactions between the engagement factors identified in this study including usability (ease of use), reminders, embedded support, rewards and goal management. Table 3 provides a summary of recommendations to help app developers and commissioners design interventions to increase effective engagement. These factors are structured around the COM-B and TDF.

Table 3. Recommendations for policy makers, industry, healthcare providers and app developers for maximising 
engagement with health and wellbeing smartphone apps.

COM-B ${ }^{\mathrm{a}}$ component

1. Capability

1. Opportunity

1. Motivation
Recommendations for policy makers, health app portal providers, app developers

1. Provide user guidance on how to use an app, visual and/or numerical summary of progress and evidence-based additional health information related to the behaviour targeted by the app

2. Minimise time required to use app where possible

3. Provide customisable reminders that users could opt out

4. Provide the option of self-monitoring features

5. Promote safety-netting and relapse prevention features such as the possibility to restart or reengage with the app later

6. Promote a routine for engagement with an app e.g. highlighting the role that routine may play in effectiveness of an app

1. Collaborate with interaction design experts and end-users to enhance the aesthetics of apps

2. Provide the possibility for community networking within the app and linking to social media as an optional feature to share progress where appropriate

3. Offer the possibility for social competition and challenges where appropriate

4. Consider the provision of embedded professional support, and if this is not feasible, providing offline one-to-one support with the uptake of and the engagement with health apps. This may improve motivational factors, such as commitment, self-confidence and perceived competence of engaging with a health app

5. We advise that exploration should be made for where engagement enhancement could be made with appropriate and proportionate machine learning and artificial intelligence or other forms of learning system.

1. Develop a time-efficient app that would require as much engagement as is required to achieve the desired outcome. This might be different for different behaviours

2. Include reinforcement in forms of feedback, encouraging messages and rewards

3. Offer intangible rewards, such as certificates or badges

4. Offer tangible rewards that can be converted as discount in other places (e.g. health insurance providers or pharmacies, sports parks)

5. Include goal setting as well as action planning features on how to achieve set goals (when applicable)

6. Take into account user's emotions about certain features by involving users in the development and update of health apps as lack of some features could provoke strong negative emotions such as disappointment and might lead to rapid disengagement

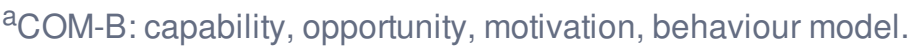

\section{Conclusion}

People perceive their capability to engage with an app as an important influence on their sustained engagement with it. This perception was inferred from people's desire for apps to contain clear user guidance, require less cognitive load and support easy self-monitoring. Tailored technology and peer and professional support may influence users' opportunity to engagement with an app and goal setting with action planning may play a key role in motivation to engage with an app.

Ethical approval: The Faculty of Medicine and Health Sciences Ethics Committee at the University of East Anglia approved this study (Reference number: 201819 - 089). The collected data is stored following the European Union General Data Protection Regulation (GDPR) and the University of East Anglia Research Data Management Policy. The data was anonymised, and all personal identifiers were removed. All participants read the participant information sheet and provided consent prior taking part in this study.

Contributorship: DS, FN, AJ, TC and JB conceptualised the study design. DS wrote the study protocol with contributions from FN, AJ, TC, JB. All authors commented on the topic guide. DS conducted stakeholder communication with PPI representatives and PHE. DS undertook recruitment of participants, data collection, data analysis, interpretation and report writing. OP double coded a proportion of the transcripts. DS, OP and FN finalised the final thematic framework. DS prepared the manuscript. All authors read, commented and contributed on the final manuscript. 
Acknowledgements: We would like to thank to all participants for their contribution to the study. The authors would like to acknowledge the support on the development of topic guide received from the patient and public representatives, the (Deputy Director of PHE Digital, the PHE Strategy and Planning and PHE Strategy and Innovation lead. Furthermore, the authors are grateful to the University College London Tobacco and Alcohol Research Group for their expert opinion on the data analysis.

\section{References}

1. World Health Organisation. Global status report on noncommunicable diseases. 2014. https://www.who.int/nmh/publications/ncd-status-report-2014/en/ (accessed June 2020).

2. Buttery AK, Mensink GBM, Busch MA. Healthy behaviours and mental health: findings from the German Health Update (GEDA). European Journal of Public Health 2014;25(2):219-25 doi: 10.1093/eurpub/cku094.

3. Vaingankar JA, Chong SA, Abdin E, et al. Understanding the relationships between mental disorders, self-reported health outcomes and positive mental health: findings from a national survey. Health and Quality of Life Outcomes 2020;18(1):55 doi: 10.1186/s12955-020-01308-0.

4. Klasnja P, Pratt W. Healthcare in the pocket: mapping the space of mobile-phone health interventions. Journal of biomedical informatics 2012;45(1):184-98 doi: 10.1016/j.jbi.2011.08.017. PMC3272165.

5. Flores Mateo G, Granado-Font E, Ferre-Grau C, Montana-Carreras X. Mobile Phone Apps to Promote Weight Loss and Increase Physical Activity: A Systematic Review and Meta-Analysis. J Med Internet Res 2015;17(11):e253 doi: 10.2196/jmir.4836. PMC4704965.

6. Schoeppe S, Alley S, Van Lippevelde W, et al. Efficacy of interventions that use apps to improve diet, physical activity and sedentary behaviour: a systematic review. International Journal of Behavioral Nutrition \& Physical Activity 2016;13(1):127.

7. Coughlin SS, Whitehead M, Sheats JQ, Mastromonico J, Smith S. A Review of Smartphone Applications for Promoting Physical Activity. Jacobs journal of community medicine 2016;2(1):021.

8. Nour M, Chen J, Allman-Farinelli M. Efficacy and External Validity of Electronic and Mobile Phone-Based Interventions Promoting Vegetable Intake in Young Adults: Systematic Review and Meta-Analysis. Journal of Medical Internet Research 2016;18(4):e58.

9. Lee S, Lindquist R. A review of technology-based interventions to maintain weight loss. Telemedicine Journal \& EHealth 2015;21(3):217-32.

10. Sherifali D, Nerenberg KA, Wilson S, et al. The Effectiveness of eHealth Technologies on Weight Management in Pregnant and Postpartum Women: Systematic Review and Meta-Analysis. Journal of Medical Internet Research 2017;19(10):e337.

11. Choo CC, Burton AAD. Mobile Phone Apps for Behavioral Interventions for At-Risk Drinkers in Australia: Literature Review. JMIR Mhealth Uhealth 2018;6(2):e18 doi: 10.2196/mhealth.6832.

12. Husain I, Spence D. Can healthy people benefit from health apps? BMJ : British Medical Journal 2015;350:h1887 doi: 10.1136/bmj.h1887. 
13. Coughlin SS, Jacobs M, Thind H, et al. On the Need for Research-Tested Smartphone Applications for Reducing Exposures to Known or Suspected Breast Carcinogens in Work and Home Environments. Journal of environment and health sciences 2015;1(4):10.15436/2378-6841.15.e004 doi: 10.15436/2378-6841.15.e004.

14. Weisel KK, Fuhrmann LM, Berking M, Baumeister H, Cuijpers P, Ebert DD. Standalone smartphone apps for mental health—a systematic review and meta-analysis. npj Digital Medicine 2019;2(1):118 doi: 10.1038/s41746-019-0188-8.

15. Perski O, Blandford A, West R, Michie S. Conceptualising engagement with digital behaviour change interventions: a systematic review using principles from critical interpretive synthesis. Transl Behav Med 2017;7(2):254-67 doi:

10.1007/s13142-016-0453-1. PMC5526809.

16. Kim Y, Oh B, Shin H-Y. Effect of mHealth With Offline Antiobesity Treatment in a Community-Based Weight Management Program: Cross-Sectional Study. JMIR Mhealth Uhealth 2020;8(1):e13273 doi: 10.2196/13273.

17. Baumel A, Muench F, Edan S, Kane JM. Objective User Engagement With Mental Health Apps: Systematic Search and Panel-Based Usage Analysis. J Med Internet Res 2019;21(9):e14567 doi: 10.2196/14567.

18. Appboy. Mobile Customer Retention Report. 2016. https://www.braze.com/blog/app-customer-retention-spring-2016report (accessed June 2020).

19. Szinay D, Jones A, Chadborn T, Brown J, Naughton F. Influences on the Uptake of and Engagement With Health and Well-Being Smartphone Apps: Systematic Review. J Med Internet Res 2020 doi: 10.2196/17572.

20. Crane D, Garnett C, Brown J, West R, Michie S. Behavior change techniques in popular alcohol reduction apps: content analysis. Journal of medical Internet research 2015;17(5):e118.

21. Lyzwinski LN, Caffery LJ, Bambling M, Edirippulige S. Consumer perspectives on mHealth for weight loss: a review of qualitative studies. J Telemed Telecare 2018;24(4):290-302 doi: 10.1177/1357633×17692722.

22. Hickey E, McMillan B, Mitchell C. Practitioners should embrace, not ignore, health apps. BMJ : British Medical Journal 2015;350:h2336 doi: 10.1136/bmj.h2336.

23. Donker T, Petrie K, Proudfoot J, Clarke J, Birch M-R, Christensen H. Smartphones for Smarter Delivery of Mental Health Programs: A Systematic Review. J Med Internet Res 2013;15(11):e247 doi: 10.2196/jmir.2791.

24. Perski O, Baretta D, Blandford A, West R, Michie S. Engagement features judged by excessive drinkers as most important to include in smartphone applications for alcohol reduction: A mixed-methods study. DIGITAL HEALTH 2018:4:2055207618785841 doi: 10.1177/2055207618785841.

25. Michie S, Atkins L, West R. The Behaviour Change Wheel: A Guide to Designing Intreventions. London: Silverback Publishing, 2014.

26. Atkins L, Francis J, Islam R, et al. A guide to using the Theoretical Domains Framework of behaviour change to investigate implementation problems. 2017;12(1):77 doi: 10.1186/s13012-017-0605-9.

27. Tong A, Sainsbury P, Craig J. Consolidated criteria for reporting qualitative research (COREQ): a 32-item checklist for interviews and focus groups. International journal for quality in health care : journal of the International Society for Quality in Health Care 2007;19(6):349-57 doi: 10.1093/intqhc/mzm042.

28. Tong A, Sainsbury P, Craig J. Consolidated criteria for reporting qualitative research (COREQ): a 32-item checklist for interviews and focus groups. International Journal for Quality in Health Care 2007;19(6):349-57 doi:

10.1093/intqhe/mzm042. 
29. Whitaker C, Stevelink S, Fear N. The Use of Facebook in Recruiting Participants for Health Research Purposes: A Systematic Review. Journal of medical Internet research 2017;19(8):e290-e90 doi: 10.2196/jmir.7071.

30. Benoot C, Hannes K, Bilsen J. The use of purposeful sampling in a qualitative evidence synthesis: A worked example on sexual adjustment to a cancer trajectory. BMC medical research methodology 2016;16(1):21 doi: 10.1186/s12874-0160114-6.

31. Ritchie J, Lewis J, Nicholls CM, Ormston R. Qualitative research practice: A guide for social science students and researchers: sage, 2013.

32. Givn LM. The SAGE Encyclopedia of Qualitative Research Methods. 2008 doi: 10.4135/9781412963909.

33. Birt L, Scott S, Cavers D, Campbell C, Walter F. Member Checking: A Tool to Enhance Trustworthiness or Merely a Nod to Validation? Qualitative Health Research 2016;26(13):1802-11 doi: 10.1177/1049732316654870.

34. Baumeister H, Reichler L, Munzinger M, Lin J. The impact of guidance on Internet-based mental health interventions A systematic review. Internet Interventions 2014;1(4):205-15 doi: https://doi.org/10.1016/j.invent.2014.08.003.

35. Perski O, Blandford A, Ubhi HK, West R, Michie S. Smokers' and drinkers' choice of smartphone applications and expectations of engagement: a think aloud and interview study. BMC Medical Informatics and Decision Making 2017;17(1):25 doi: 10.1186/s12911-017-0422-8.

36. Verma M, Hontecillas R, Tubau-Juni N, Abedi V, Bassaganya-Riera J. Challenges in Personalized Nutrition and Health. Frontiers in Nutrition 2018;5(117) doi: 10.3389/fnut.2018.00117.

37. Franco RZ, Fallaize R, Lovegrove JA, Hwang F. Popular Nutrition-Related Mobile Apps: A Feature Assessment. JMIR Mhealth Uhealth 2016;4(3):e85 doi: 10.2196/mhealth.5846. PMC4985610.

38. Perski O, Crane D, Beard E, Brown J. Does the addition of a supportive chatbot promote user engagement with a smoking cessation app? An experimental study. DIGITAL HEALTH 2019;5:2055207619880676 doi: $10.1177 / 2055207619880676$.

39. Yardley L, Spring BJ, Riper H, et al. Understanding and Promoting Effective Engagement With Digital Behavior Change Interventions. Am J Prev Med 2016;51(5):833-42 doi: 10.1016/j.amepre.2016.06.015.

40. Mitchell M, White L, Oh P, et al. Uptake of an Incentive-Based mHealth App: Process Evaluation of the Carrot Rewards App. JMIR Mhealth Uhealth 2017;5(5):e70 doi: 10.2196/mhealth.7323.

41. Lerner JS, Li Y, Valdesolo P, Kassam KS. Emotion and Decision Making. Annual Review of Psychology 2015;66(1):799-823 doi: 10.1146/annurev-psych-010213-115043.

42. Kohl LF, Crutzen R, de Vries NK. Online prevention aimed at lifestyle behaviors: a systematic review of reviews. J Med Internet Res 2013;15(7):e146 doi: 10.2196/jmir.2665. PMC3714003. 